\title{
Genetic basis of negative heterosis for growth traits in chickens revealed by genome-wide gene expression pattern analysis
}

Chunning Mai ${ }^{\dagger}$, Chaoliang Wen ${ }^{\dagger} \mathbb{B}$, Zhiyuan Xu, Guiyun Xu, Sirui Chen, Jiangxia Zheng, Congjiao Sun ${ }^{*}$ and Ning Yang ${ }^{*}$ (D)

\begin{abstract}
Background: Heterosis is an important biological phenomenon that has been extensively utilized in agricultural breeding. However, negative heterosis is also pervasively observed in nature, which can cause unfavorable impacts on production performance. Compared with systematic studies of positive heterosis, the phenomenon of negative heterosis has been largely ignored in genetic studies and breeding programs, and the genetic mechanism of this phenomenon has not been thoroughly elucidated to date. Here, we used chickens, the most common agricultural animals worldwide, to determine the genetic and molecular mechanisms of negative heterosis.

Results: We performed reciprocal crossing experiments with two distinct chicken lines and found that the body weight presented widely negative heterosis in the early growth of chickens. Negative heterosis of carcass traits was more common than positive heterosis, especially breast muscle mass, which was over $-40 \%$ in reciprocal progenies. Genome-wide gene expression pattern analyses of breast muscle tissues revealed that nonadditivity, including dominance and overdominace, was the major gene inheritance pattern. Nonadditive genes, including a substantial number of genes encoding ATPase and NADH dehydrogenase, accounted for more than $68 \%$ of differentially expressed genes in reciprocal crosses (4257 of 5587 and 3617 of 5243, respectively). Moreover, nonadditive genes were significantly associated with the biological process of oxidative phosphorylation, which is the major metabolic pathway for energy release and animal growth and development. The detection of ATP content and ATPase activity for purebred and crossbred progenies further confirmed that chickens with lower muscle yield had lower ATP concentrations but higher hydrolysis activity, which supported the important role of oxidative phosphorylation in negative heterosis for growth traits in chickens.
\end{abstract}

Conclusions: These findings revealed that nonadditive genes and their related oxidative phosphorylation were the major genetic and molecular factors in the negative heterosis of growth in chickens, which would be beneficial to future breeding strategies.

Keywords: Chicken, Growth, Gene expression patterns, Heterosis, Oxidative phosphorylation

\footnotetext{
*Correspondence: cjsun@cau.edu.cn; nyang@cau.edu.cn

${ }^{\dagger}$ Chunning Mai and Chaoliang Wen contributed equally to this work.

National Engineering Laboratory for Animal Breeding and Key Laboratory of

Animal Genetics, Breeding and Reproduction, Ministry of Agriculture and

Rural Affairs, China Agricultural University, Beijing 100193, China
}

(C) The Author(s). 2021 Open Access This article is licensed under a Creative Commons Attribution 4.0 International License, which permits use, sharing, adaptation, distribution and reproduction in any medium or format, as long as you give appropriate credit to the original author(s) and the source, provide a link to the Creative Commons licence, and indicate if changes were made. The images or other third party material in this article are included in the article's Creative Commons licence, unless indicated otherwise in a credit line to the material. If material is not included in the article's Creative Commons licence and your intended use is not permitted by statutory regulation or exceeds the permitted use, you will need to obtain permission directly from the copyright holder. To view a copy of this licence, visit http://creativecommons.org/licenses/by/4.0/ The Creative Commons Public Domain Dedication waiver (http://creativecommons.org/publicdomain/zero/1.0/) applies to the data made available in this article, unless otherwise stated in a credit line to the data. 


\section{Background}

Heterosis, first proposed by Shull in 1908 [1], is defined as the deviation between $F_{1}$ reciprocal crosses and their parental lines mean [2]. Heterosis has become a routine strategy for livestock and crop breeding and has driven great improvements in performance or livability over the last century. The fundamental mechanism underlying heterosis will determine whether it can be manipulated for the benefit of agriculture and biotechnology. Numerous studies have attempted to explain the genetic mechanism of heterosis, and three classic quantitative genetic hypotheses have been proposed: dominance [3, 4], overdominance $[1,5]$, and epistasis $[6,7]$. However, these three models were mainly theoretical and could not provide a full explanation for the molecular basis and physiological causes of heterosis $[8,9]$.

At the molecular level, variation in gene expression is thought to constitute a significant source of phenotypic diversity [10]. Investigation of differentially expressed genes (DEGs) between crossbred and their parent lines might contribute to improving our understanding of the molecular basis for heterosis. In particular, the gene expression patterns involved in some metabolic pathways are obviously correlated with heterotic phenotypes. For example, several studies on heterosis in rice revealed that differentially expressed genes between hybrids and their parents were involved in energy metabolism, which contributed in a significant way to the increased yield of hybrids [11, 12]. Fujimoto et al. [13] demonstrated that the higher photosynthetic efficiency of Arabidopsis hybrids was obviously associated with the early increase in the activity of genes involved in chlorophyll biosynthesis and photosynthesis, which contributed to increased heterotic biomass. Similar studies were also reported in animals. Hedgecock et al. [14] conducted transcriptomic analysis in Crassostrea gigas and found that nonadditive genes and their related protein metabolism played important roles in growth heterosis.

In addition to positive heterosis, negative heterosis is also observed in many farm animals [15-19] and plants [20-23]. Negative heterosis can cause unfavorable impacts on production performance in agriculture, such as negative heterosis of body weight in quails $[15,16]$, carcass traits in beefs [17], hybrid necrosis in plants $[20,21]$, and hybrid weakness of shoot dry weight [22] and height [23] in rice. The poultry industry has a long history of using crosses between different populations to take advantage of strain complementarity. In theory, the magnitude of heterosis is inversely correlated to the extent of genetic similarity between parental lines, and interspecific crosses show greater heterosis than intraspecific crosses [24]. However, abundant evidence has revealed the existence of negative heterosis for growth traits in chickens when the genetic background of the parents varies greatly. Williams et al. [25] used the high- and low-body-weight chicken lines from Virginia Tech for heterosis analysis, and approximately $-24 \%$ and $-16 \%$ heterosis was observed for the body weights of reciprocal crosses at 4 and 8 weeks of age, respectively. Similar results were reported by Jull and Quinn [26], Maw [27], Liu et al. [28], and Sutherland et al. [29]. Negative heterosis for carcass performance, especially for muscle mass in reciprocal crosses, was reported by Sun et al. [30] when broiler and layer chickens were used as parents.

However, the genetic and molecular basis of heterosis for growth traits in chickens is still a mystery. Chickens are the most common and widespread domestic animals worldwide, as well as a great source of meat for humans. Revealing the genetic mechanisms of negative heterosis for growth traits will improve yield to meet the chicken meat demands of humans worldwide. Breast muscle is the largest proportion of body weight and is an important indicator of the growth rate in chickens [31]; thus, investigating the negative heterosis of breast muscle mass can be a breakthrough to explore this unclear phenomenon. The development of transcriptome sequencing technologies has allowed unbiased and reproducible sequencing of whole transcriptomes, which are valuable for characterizing the patterns of gene expression and have been used to unravel the mechanisms of heterosis [32-34]. In the current study, Cornish $(C)$ and Rhode Island White $(R)$ were used as parental lines to produce the $F_{1}$ generation. Cornish, as a standard broiler breed, has been selected for growth and, in particular, for muscle growth. Rhode Island White, a layer breed, has been intensively selected for egg production. The RNAsequencing strategy was used to identify the transcriptomic differences in the breast muscle of reciprocal crosses and their parental lines. The objective of this study was to provide new insight into the molecular basis of negative heterosis for growth performance in chickens.

\section{Methods}

\section{Experimental populations}

Two domesticated chicken breeds, Cornish (meat-type chicken, C line) and Rhode Island White (egg-type chicken, $\mathrm{R}$ line), from Beijing Huadu Yukou Poultry Industry Co., Ltd. were employed as parents in this study to produce purebred progenies and reciprocal crosses (Fig. 1a). The $\mathrm{C}$ line has been selected for 42day body weight for 7 generations, whereas the $\mathrm{R}$ line has been intensively selected for total egg production to 300 days of age for 15 generations. We selected 10 


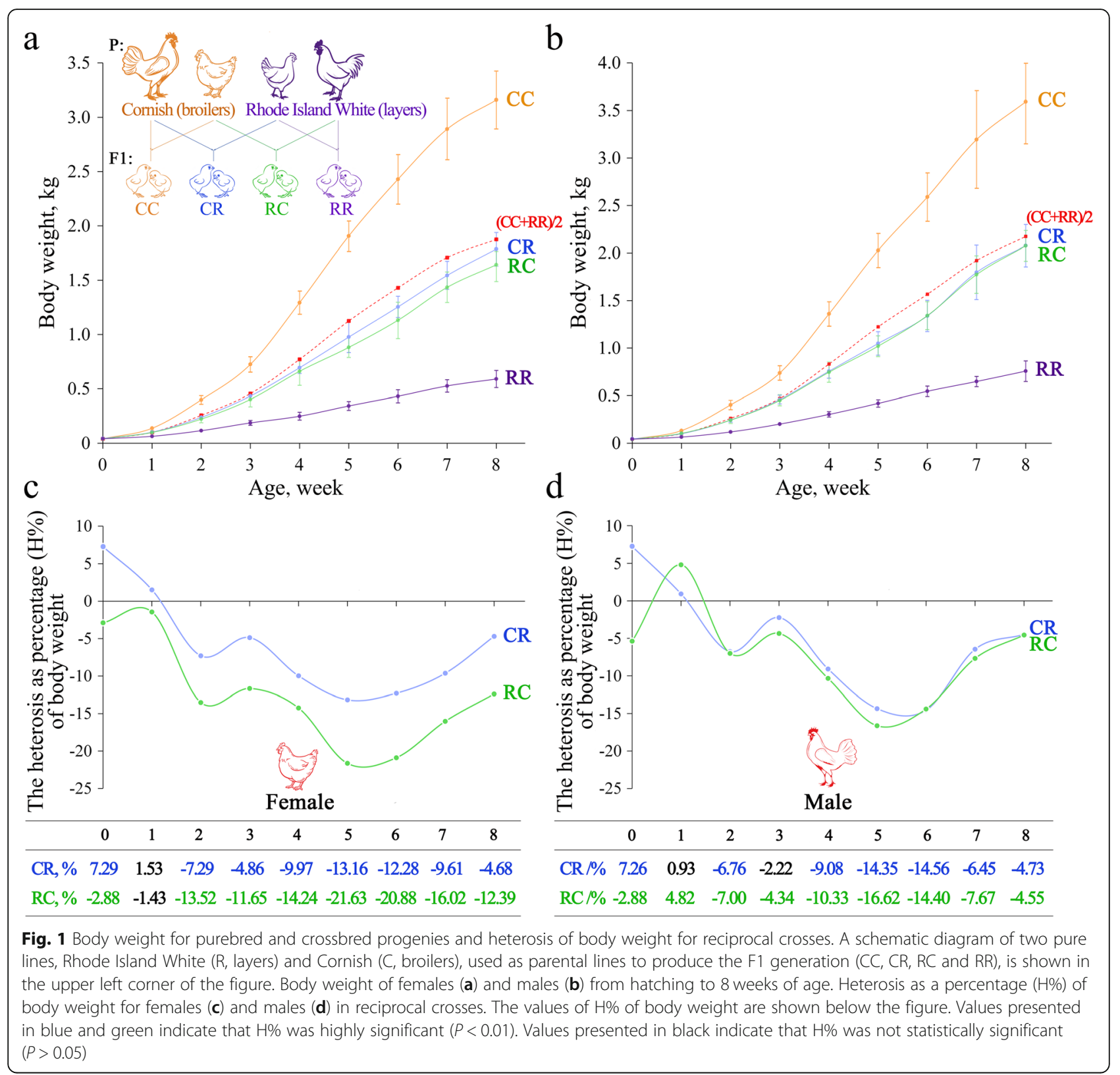

males and 120 females from the 8th generation of the $\mathrm{C}$ line and 10 males and 80 females from the 16th generation of the $\mathrm{R}$ line as parents according to the following criteria: (i) cocks in each line with similar body weight and good semen quality and (ii) hens in each line with similar body weight and high egg production. These chickens were housed with individual cages in the same poultry facility. Each male (both $\mathrm{C}$ and $\mathrm{R}$ ) was mated with $6 \mathrm{C}$ and $4 \mathrm{R}$ females by artificial insemination. The eggs were collected and recorded daily. Finally, a total of 632 chicks (347 for females and 285 for males) with clear pedigree information were hatched on the same day and used for subsequent studies.
Phenotypic measurement and sample collection

At hatching, chicks were identified as males and females by vent sexing and then reared in separated cages under the same environment with free access to feed and water. The hatched chicks were wing-banded for individual identification. The four genetic combinations were reared in different cages to eliminate size disparities and reduce competition. The body weights were measured weekly from hatch to 8 weeks of age (Table S1). The length of the left shank and sternum for chickens were measured at 3, 6, and 8 weeks of age (Table S2). At 6 weeks of age, we randomly selected 64 female chickens (14, 14, 16 and 20 for CC, RR, CR and RC, respectively) and 44 male chickens $(10,9,15$ and 10 for CC, RR, CR 
and $\mathrm{RC}$, respectively) from different half-sib families. These chickens were euthanized by cervical dislocation, and the following carcass traits were measured by an electronic balance: slaughter weight (measured after bloodletting), both left and right wing weight, breast muscle weight (pectoralis major and minor), drumstick weight (bone and muscle), and drumstick bone weight. The drumstick muscle weight was calculated as the drumstick weight subtracted from the drumstick bone weight. Heterosis as a percentage $(\mathrm{H} \%)$ of the abovementioned traits was calculated according to the following equation:

$$
H \%=\frac{\overline{F_{1}}-\left(\overline{P_{M}}+\overline{P_{F}}\right) / 2}{\left(\overline{P_{M}}+\overline{P_{F}}\right) / 2} \times 100 \%
$$

where $\overline{F_{1}}, \overline{P_{M}}$ and $\overline{P_{F}}$ are the mean phenotypes of the reciprocal crosses, the maternal and paternal lines, respectively. In order to evaluate the significance of $\mathrm{H} \%$, Student's t-value was estimated based on the formula of Wu et al. [35]:

$$
\mathrm{t}=\frac{H \%}{2 \sqrt{\frac{\sum\left(F_{1 i}-\overline{F_{1}}\right)^{2}}{N-1} /\left[\left(\overline{P_{M}}+\overline{P_{F}}\right) \times \sqrt{N}\right]}}
$$

where $F_{1 i}$ is the phenotype of individual $i$ from reciprocal crosses; $N$ is the number of birds in RC or CR. We obtained the $P$-value using the pt. function in the $\mathrm{R}$ program (https://www.r-project.org/) according to the $t$ value and the degrees of freedom. $\mathrm{H} \%$ was considered significant and highly significant if $P$-value $<0.05$, and $P$ value $<0.01$, respectively. Meanwhile, six female offspring (from 4-6 half-sib families) of each group, except for five female offspring in the RC group, were selected, and the left pectoralis major muscle of these chickens was isolated for subsequent RNA sequencing.

\section{RNA extraction and sequencing}

Total RNA was extracted using the TRIzol $^{\circ}$ Reagent (Invitrogen, USA) according to the manufacturer's instructions and then dissolved in DEPC-treated water. To ensure that RNA was isolated successfully, the extracted RNA was first evaluated by $1 \%$ agarose gel electrophoresis. Then, the RNA purity, concentration and integrity of all eligible RNA extraction were determined by a NanoPhotometer ${ }^{\circ}$ spectrophotometer (IMPL EN, CA, USA), a Qubit ${ }^{\circ} 2.0$ Fluorimeter (Life Technologies, CA, USA), and an RNA Nano 6000 Assay Kit from the Bioanalyzer 2100 system (Agilent Technologies, CA, USA), respectively. The samples with an RNA integrity number value that greater than 7.0 were considered as high-quality RNA samples. A total of 23 samples were qualified for RNA sequencing library construction.
Approximately $3 \mu \mathrm{g}$ of RNA per sample was subjected to RNA-seq library construction using the NEBNext ${ }^{\circ}$ UltraTM RNA Library Prep Kit (Illumina, USA) according to the manufacturer's guide. After PCR amplification and purification, $150 \mathrm{bp}$ paired-end sequencing was performed on the Illumina Hiseq $\mathrm{X}$ Ten platform (Illumina Inc., San Diego, CA, USA) and generated in nearly 750.13 million raw reads.

\section{Quality control and mapping}

To minimize mapping errors, reads that met the following parameter were removed: a) containing adaptors; b) with more than $10 \%$ unknown nucleotides; c) with more than 50\% low-quality bases (Qphred $\leq 20$ ). The chicken reference genome (galGal5) and gene model annotation files were downloaded from the Ensembl database (ftp:// ftp.ensembl.org/pub/release-91/). After quality control, over 721.42 million high quality reads with Q20 > 95\% (Table S3) were aligned to the chicken reference genome using Hisat2 (v2.0.5) [36]. Approximately $76 \%$ of the high quality reads in each sample were mapped to the reference genome. Over $80 \%$ of reads were assigned to exonic regions, approximately $4 \%$ were assigned to intronic regions, and $16 \%$ were assigned to intergenic regions.

\section{Differential gene expression analyses}

The mapped reads of each sample were assembled by StringTie (v.1.3.3b) [37]. The function of novel genes was annotated based on the Pfam database (v.31.0) [38]. Then, the gene count matrix table was generated by featureCounts (v1.5.0-p3) [39]. FPKM (fragments per kilobase million) values were extracted from the StringTie outputs. To enhance the statistical power for DEGs, the genes with an average FPKM $<1$ were removed. Meanwhile, the sex-linked genes were removed from the following analysis. After these steps, 11,050 genes were filtered, and the remaining 11,544 genes were used for differential expression analysis between two purebred lines (CC vs. RR) and between reciprocal crosses and purebred lines $(\mathrm{CR}$ vs. $\mathrm{CC}, \mathrm{CR}$ vs. RR, RC vs. $C C$ and $R C$ vs. RR) using the DESeq2 package (v.1.16.1) [40] in $\mathrm{R}$ project. We presented DESeq2, a method for differential analysis of count data, using the empirical Bayes shrinkage method to estimate dispersions and fold changes. The $P$-value was calculated by the Wald test. To control the false discovery rate, the resulting $P$-values were adjusted for multiple testing using the Benjamini-Hochberg method. Genes with an adjusted $P$-value $<0.05$ were considered differentially expressed genes in the corresponding comparison. 


\section{Evaluation of differential inheritance patterns}

We used the average FPKM value of each group and the adjusted $P$-value to evaluate different inheritance patterns of genes (Table S4) [32]. These genes were further classified into three inheritance patterns: additivity, dominance and overdominance, based on the level of gene expression exhibited by reciprocal crosses and parental lines. In brief, additivity (I and XII) occurred when the gene expression was significantly different between the two parental lines (adjusted $P$-value $<0.05$ ), and the gene expression of reciprocal crosses ( $\mathrm{CR}$ or $\mathrm{RC}$ ) was higher than one parental line but lower than the other parental line. Gene expression within CR/RC that was not significantly different from one parental line but significantly higher (or lower) than the other parental line was regarded as dominance (II, IV, IX, and XI). Gene expression within $\mathrm{CR} / \mathrm{RC}$ that was significantly higher (or lower) than both parental lines (CC and RR) was considered overdominance (V, VI, VIII, III, VII, and X).

To confirm the reliable of gene expression patterns reveled by RNA-seq, we performed quantitative realtime PCR (qRT-PCR) experiments. qRT-PCR reactions were performed with three technical replicates for each individual. The details of qRT-PCR and related results have been previously described [34].

\section{GO enrichment and KEGG pathway analyses}

To investigate the biological function of nonadditive genes involved, we performed functional enrichment analysis, including Gene Ontology (GO) categories and Kyoto Encyclopedia of Genes and Genomes (KEGG) pathways, using the Clusterprofile package [41] in the $\mathrm{R}$ project. The GO terms and KEGG pathways with FDR $<0.05$ (BH method) were considered significant.

\section{ATP content assay}

ATP content was detected using an ATP assay kit (S0026B, Beyotime Biotechnology, China) as described in a previous study [42]. The method is based on the theory that luciferase catalyzes luciferin to form fluorescence, which requires energy provided by ATP. Thus, the emitted fluorescence intensity is linearly related to the ATP concentration. Briefly, tissue samples $(20 \mathrm{mg})$ were homogenized on ice with $150 \mu \mathrm{L}$ of ice-cold assay buffer. It was then centrifuged at $12,000 \mathrm{r} / \mathrm{min}$ for 10 $\min$ at $4{ }^{\circ} \mathrm{C}$ to remove insoluble materials, and the supernatant was collected. An aliquot $(100 \mu \mathrm{L})$ of ATP detection working solution was added to each well of a white 96-well plate. After incubation for $3 \mathrm{~min}$ at room temperature, $50 \mu \mathrm{L}$ of supernatant was added to the wells. Luminescence was measured by a fluorescence microplate reader.

\section{ATPase activity assay}

ATPase activity was assessed using an ATPase activity assay kit (MAK113, Millipore Sigma, St. Louis, MO, USA) according to the manufacturer's instructions. ATPase hydrolyzes ATP into ADP and free phosphate. Free phosphate causes the malachite green reagent to form a stable dark green colorimetric product that is proportional to the ATPase activity. In brief, tissue samples (20 mg) were homogenized on ice with $200 \mu \mathrm{L}$ of ice-cold assay buffer. They were then centrifuged at $14,000 \mathrm{r} / \mathrm{min}$ for $10 \mathrm{~min}$ at $4{ }^{\circ} \mathrm{C}$ to remove insoluble materials, and the supernatant was collected. An aliquot $(30 \mu \mathrm{L})$ of the reaction mixture solution was added to each well of a 96-well flat-bottom plate and incubated for $30 \mathrm{~min}$ at room temperature. Then, $200 \mu \mathrm{L}$ of reagent was added to each well and incubated for an additional $30 \mathrm{~min}$ at room temperature to terminate the enzyme reaction. Finally, the absorbance was determined at $620 \mathrm{~nm}$ for all samples.

\section{Statistical analysis}

Differences in breast muscle weight, ATP content and ATPase activity among parental lines and reciprocal crosses were assessed using ANOVA followed by Tukey's HSD test in the $\mathrm{R}$ program. The results were considered to be statistically significant when the adjusted $P$-value was less than 0.05 .

\section{Results}

Negative Heterosis of body weights and carcass traits

As described in Fig. 1a, we chose the $\mathrm{C}$ and $\mathrm{R}$ breeds to produce purebred (CC and RR) and reciprocal crossbred progenies (RC and $\mathrm{CR}$ ). The body weight of each progeny was measured weekly from hatching to 8 weeks of age. The traits of shank length and sternum length were measured at 3, 6, and 8 weeks of age. The correlation among body weights at different ages for females and males varied from 0.115 to 0.991 and from 0.012 to 0.991, respectively (Fig. S1). As shown in the dynamic growth of parental lines and reciprocal crosses, the body weights of females and males in CR and RC from 2 to 8 weeks of age were lower than the average of $C C$ and $R R$ (Fig. 1a, b and Table S1), although reciprocal crosses exhibited slightly positive heterosis for the length of shank and sternum at 6 and 8 weeks of age (Table S5). The degree of heterosis for body weight is displayed in Fig. 1c and $\mathrm{d}$ in terms of heterosis as a percentage. The $\mathrm{H} \%$ of body weight varied from $-21.63 \%$ to $7.29 \%$ and from $-16.62 \%$ to $7.26 \%$ for females and males, respectively. The negative heterosis of females and males reached a maximum value between the fifth and sixth weeks of age. In females, compared with CR (range of $-13.16 \%$ to $7.29 \%$ ), the $\mathrm{H} \%$ was smaller in $\mathrm{RC}$, which varied from $-21.63 \%$ to $-1.43 \%$. In males, compared with 
CR (range of $-14.56 \%$ to $7.26 \%$ ), the $\mathrm{H} \%$ was smaller in $\mathrm{RC}$, which varied from $-16.62 \%$ to $4.82 \%$. In CR (females) and $\mathrm{RC}$ (males), the $\mathrm{H} \%$ of body weight decreased from hatching to 5 weeks of age and showed a slight increasing trend from 5 to 8 weeks of age, while in RC (females) and $\mathrm{CR}$ (males), the inflection point was 6 weeks of age.
Given that fast growing broilers are mostly marketed at 6 weeks of age, we randomly slaughtered 108 chickens from four groups for carcass composition analysis at 42 days of age. As shown in Fig. 2 and Table S6, most carcass traits, including slaughter weight, breast muscle weight, drumstick weight, and drumstick muscle weight,
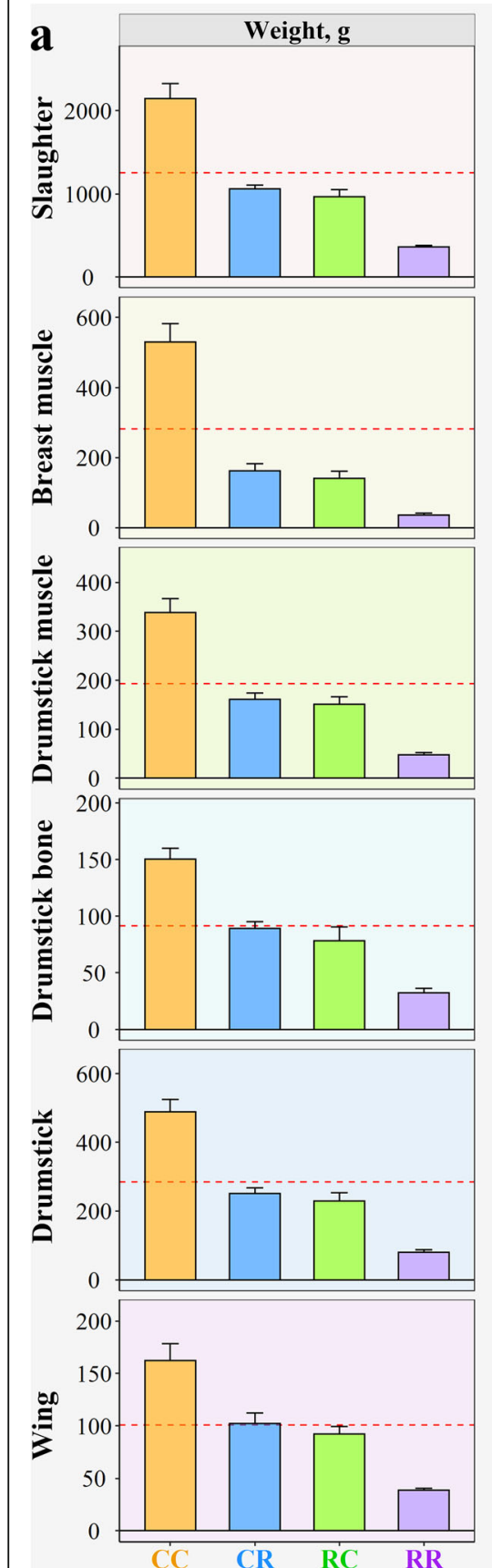
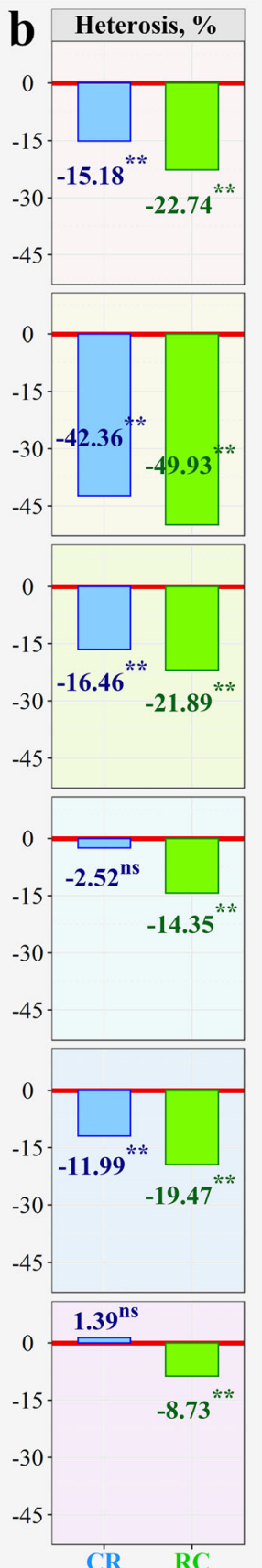


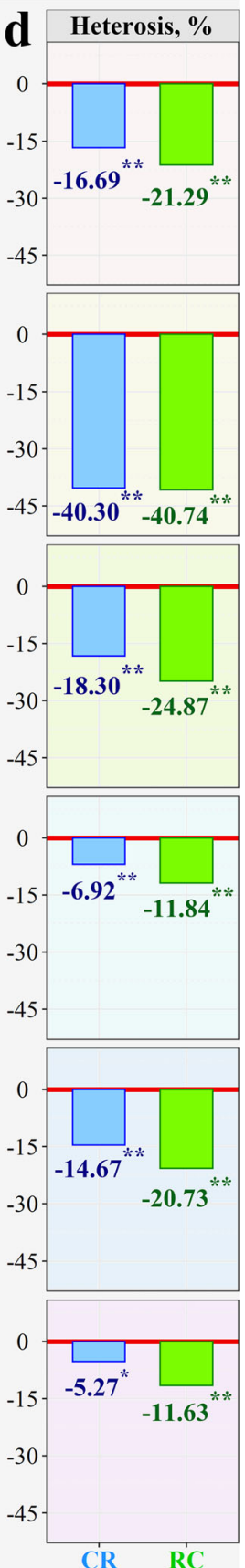

Fig. 2 Heterosis of carcass performance for purebred and crossbred progenies at 6 weeks of age. The breast muscle weight, drumstick bone weight, drumstick weight (bone and muscle) and wing weight were measured on both sides. The drumstick muscle weight was calculated as the drumstick weight subtracted from the drumstick bone weight. a-b Female. c-d Male. For (a) and (c), the dashed red line represents the midparent value. For $(\mathbf{b})$ and $(\mathbf{d}), \mathrm{ns}^{*}$ and ${ }^{* *}$ indicate that the heterosis as a percentage $(\mathrm{H} \%)$ was not statistically significant $(P>0.05)$, significant $(P<0.05)$ and highly significant $(P<0.01)$, respectively 
showed extremely significant negative heterosis $(P<$ 0.01 ) in reciprocal crosses of females and males. Among these various carcass traits, the negative heterosis of breast muscle weight was the largest, e.g., $-42.35 \%$ (CR) and $-49.93 \%$ (RC) in females and $-40.29 \%$ (CR) and $-40.75 \%(\mathrm{RC})$ in males. Meanwhile, the correlation of body weight and breast muscle weight at 6 weeks of age was 0.98 for both females and males (Fig. S2).

\section{Inheritance of gene expression in reciprocal crosses}

As noted above, the negative heterosis of body weight and carcass traits were widespread in the present study. The more fundamental question is why the reciprocal progenies exhibited this phenomenon. Thus, the transcriptional data of breast muscle tissues for the four groups were used to analyze the differences in gene expression between parental lines and reciprocal crosses. A principal component analysis (PCA) was performed to visualize the differences in gene expression. The PCA plot showed that the four groups were obviously separated from each other (Fig. 3a), indicating that there were visible differences in gene expression between the two parental lines and between the parental lines and reciprocal crosses.

A total of 6253 DEGs between the two parental lines and between the reciprocal crosses and parental lines were identified (Fig. 3b and Fig. S3), e.g., 5147 (RR vs. CC), 3205 (CR vs. CC), 1628 (CR vs. RR), 1184 (RC vs. $\mathrm{RR}$ ) and 4470 ( $\mathrm{RC}$ vs. CC). These DEGs were divided into 12 types (I, II, III, IV, V, VI, VII, VIII, IX, X, XI and XII; for details, see Table S4) based on the level of gene expression exhibited by reciprocal crosses and parental lines. The number of the 12 type genes in the CR and $\mathrm{RC}$ groups is shown in Fig. 3c and Table S7. The 12 types were further classified into 3 main inheritance patterns: additivity (I, XII), dominance (II, IV, IX and XI), and overdominance (III, V, VI, VII, VIII and X). The number of dominant genes was 3324 and 3851 in CR and $\mathrm{RC}$, respectively. The number of overdominant genes was 293 and 406 in RC and CR, respectively. Nonadditivity, including dominance and overdominace, was the major gene inheritance pattern. Nonadditive genes accounted for $68.99 \%$ and $76.20 \%$ of DEGs in CR and $\mathrm{RC}$, respectively (Fig. 3d).

\section{Nonadditive inheritance is related to oxidative phosphorylation}

Apart from the focus on gene expression patterns in reciprocal crosses, we are more interested in the biological processes that additive and nonadditive genes are related to. We tested for enrichment of these genes against GO and KEGG pathways to detect the metabolic pathways involved. The functional enrichment analyses showed no significant GO terms or pathways detected in additive genes of the RC group, although one KEGG pathway, ribosome, was significantly enriched in additive genes of the CR group. However, as shown in Fig. 4a, the dominant genes of $\mathrm{CR}$ and $\mathrm{RC}$ were both significantly enriched in $46 \mathrm{GO}$ terms, including $2 \mathrm{GO}$ terms of molecular function, $32 \mathrm{GO}$ terms of cell composition and $12 \mathrm{GO}$ terms of biological process. The majority of these GO terms were associated with mitochondrial components and energy metabolism. Furthermore, the KEGG pathway analysis showed that one shared pathway, oxidative phosphorylation, was significantly enriched in the dominant genes of the $\mathrm{CR}$ and $\mathrm{RC}$ groups (Fig. 4b). Additionally, overdominant genes of both $\mathrm{RC}$ and $\mathrm{CR}$ were also enriched in GO terms related to mitochondrial components and energy metabolism (Table S8). The oxidative phosphorylation pathway was also detected in the overdominant genes of the CR and RC groups (Fig. S4).

Given that nonadditive genes were significantly enriched in the pathway of oxidative phosphorylation, we further analyzed the nonadditive genes in the CR and $\mathrm{RC}$ groups. The number of nonadditive genes enriched in oxidative phosphorylation was 33 and 59 in the CR and $\mathrm{RC}$ groups, respectively (Fig. 5a). These genes were related to $\mathrm{NADH}$ dehydrogenase, cytochrome $\mathrm{c}$ reductase, cytochrome c oxidase, ATP synthase, ATPase and succinate dehydrogenase. Among those, 31 shared genes were detected in the CR and RC groups (Fig. 5a and Table S9). Considering that nonadditive genes contained 10 different types, we further analyzed which type was important to the process of oxidative phosphorylation and found that types IV and II were the major gene expression patterns in $\mathrm{CR}$ and $\mathrm{RC}$, respectively (Fig. 5b). Type IV in CR and type II in RC accounted for $77.42 \%$ (24 of 31 ) and $61.29 \%$ (19 of 31 ) of shared genes, respectively. It is worth noting that the expression level of these genes in reciprocal progenies biased to the $\mathrm{R}$ line, and the expression level in the $\mathrm{R}$ line was significantly higher than that in the $\mathrm{C}$ line.

\section{ATP content and ATPase activity detection}

The gene expression pattern results showed that nonadditive genes were related to the biological process of oxidative phosphorylation, implying that energy metabolism plays a vital role in negative heterosis of breast muscle. To further confirm the relationship between oxidative phosphorylation and negative heterosis for growth traits, we detected the ATP concentration and hydrolysis activity of breast muscle tissues for the CC, RR, CR, and RC groups. ATPases are a group of enzymes that catalyze the hydrolysis of ATP to form ADP. The detection of ATP content and ATPase activity revealed that the group with lower growth traits had lower ATP content but higher ATPase activity (Fig. 6a-d). As shown in Fig. 6a, breast muscle mass was significantly higher in 
a

- $\mathrm{CC} \bigcirc \mathrm{CR}$ ○ $\mathrm{RC} \quad$ ○ $\mathrm{RR}$

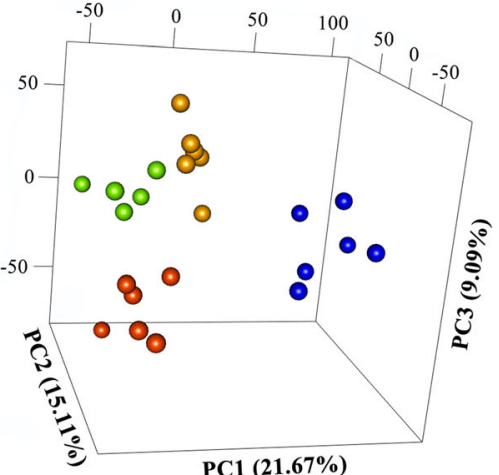

C
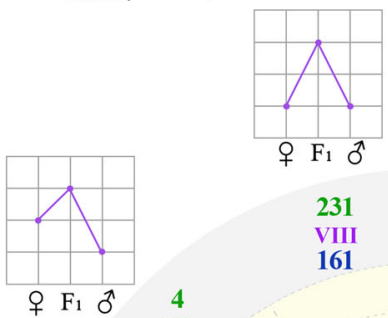

4
VI
15
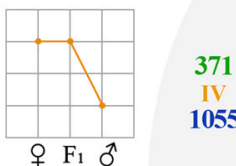

371
IV
1055

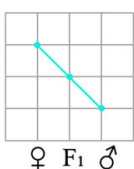

657
XII
795

우 $\mathrm{F}_{1} \mathrm{O}^{\hat{2}}$
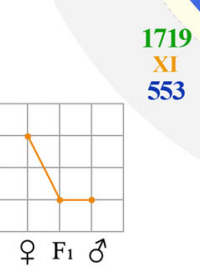

1719
$\times 1$
553

553

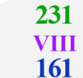

231
VIII
161

b


52

4

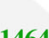

1464

11
507

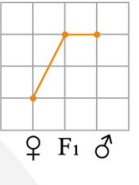

673

831
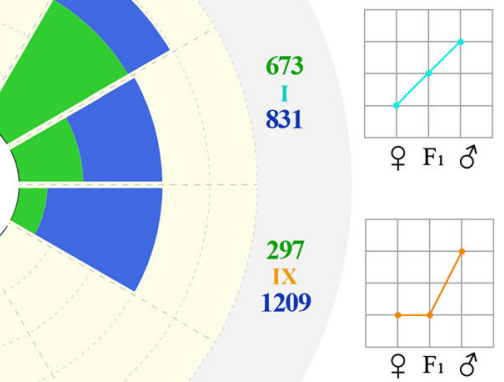

6

III
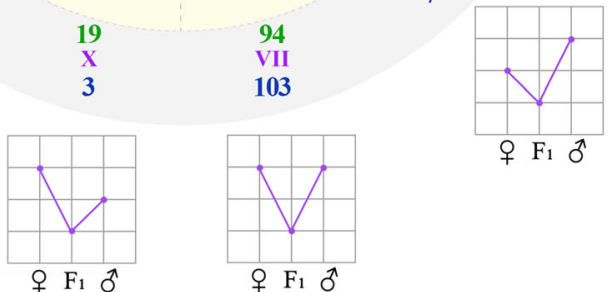

d

$5.59 \%$

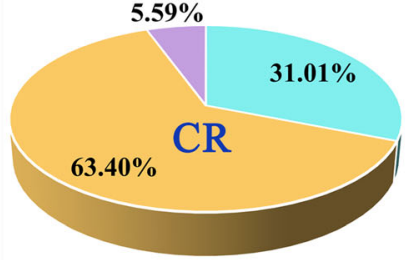

Additivity: I \& XII — Dominance: II, IX, XI \& IV
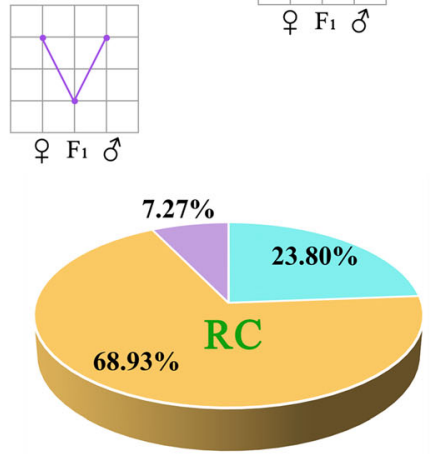

Overdominance: III, VII, X, VI, VIII \& V

Fig. 3 (See legend on next page.) 
(See figure on previous page.)

Fig. 3 Analysis of gene inheritance patterns. a Principal component analysis of the reciprocal crosses (CR, RC) and the parental lines (RR, CC). $\mathbf{b}$ The number of DEGs among $F_{1}$ progenies. $\mathbf{c}$ Inheritance patterns of DEGs between reciprocal crosses and parental lines. DEGs were divided into 12 types, e.g., class I, II, III, IV, V, VI, VII, VIIII, IX, X, XI and XII, and further classified into three inheritance patterns: additivity (class I and XII), dominance (class II, IV, IX, and XI) and overdominance (class III, V, VI, VII, VIII, and X), based on the level of gene expression exhibited by reciprocal crosses and parental lines. Additivity, dominance, and overdominance are presented in blue, orange, and purple, respectively. Each class was accompanied by diagrams representing the relative expression levels of the maternal line (left dot), $F_{1}$ (middle dot), and paternal line (right dot). The number of DEGs in each class is shown above this class (green numbers, represented as the RC group) and below (blue numbers, represented as the (R group). $\mathbf{d}$ The proportion of additive, dominant and overdominant genes in DEGs

CC than in RR, RC, and CR. The same trend was observed in body weight (Fig. 6b) and ATP content (Fig. 6c), and an opposite trend for ATPase activity is presented in Fig. 6d.

\section{Discussion}

The utilization of heterosis has contributed tremendously to the increased productivity in many domesticated animals and crops for decades. In terms of the calculation formula, $\mathrm{H} \%$ can be a positive or negative sign. Compared with the extensive studies on positive heterosis [11, 13, 43-45], the phenomenon of negative heterosis is overlooked in breeding programs and genetic studies, even though it exists widely in nature. In the present study, we observed that negative heterosis of body weight and carcass traits in juvenile chickens was more common than positive heterosis. This phenomenon was also reported by Williams et al. [25], Liu et al. [28] and Sutherland et al. [46]. Among the carcass characteristics, we found that meat production displayed the largest negative heterosis in reciprocal crosses of females and males. The $\mathrm{H} \%$ of breast and drumstick muscle weight was over $-40 \%$ and $-20 \%$, respectively. These results were consistent with previous research showing that the negative heterosis of breast muscle weight in crosses was the largest among carcass traits when using broilers and layers as parents [30]. Positive or negative heterosis does not imply superiority or inferiority since it depends on the trait's biological significance and production preference [25, 34]. In livestock production, the negative heterosis of growth and meat yield was unfavorable since it reduced the edible carcass portions. We characterized the transcriptome profiles of breast muscle in reciprocal crosses and the parental lines herein to reveal the potential mechanisms of negative heterosis for growth traits in chickens.

A large number of DEGs between reciprocal crosses and the parental lines were identified. The number of DEGs between two parental lines was greater than that between reciprocal crosses and their parental lines. This result was consistent with a previous study [11] and indicated that the genetic difference between two parental lines was larger than that between reciprocal crosses and their parental lines. Nonadditive genetic variance can result from a nonlinear phenotypic effect of alleles at one locus, as in the case of dominant or recessive allele pairs in classical genetics. Thus, the nonadditive expression pattern is critically important to the formation of heterosis [2, 14]. Recently, gene expression pattern analysis of chicken liver tissues revealed that overdominant genes related to lipid metabolism played a central role in the heterosis of fat deposition [34]. Wu et al. [33] reported that dominant genes involved in carbohydrate metabolism were associated with heterosis for body weight in Drosophila melanogaster. In the present study, we classified these DEGs between reciprocal crosses and their parental lines into additivity, dominance and overdominance. Our results revealed that nonadditivity, including dominance and overdominance, was the major gene expression pattern in reciprocal crosses. Similar results were observed in Arabidopsis [2], Crassostrea gigas [14] and chickens [34]. Previous reports in Medicago sativa [47] and Larix kaempferi [48] showed that the proportion of nonadditive genes in heterotic hybrids was higher than that in nonheterotic hybrids. It should be noted that nonadditive genes accounted for $76 \%$ of DEGs in the RC group, which was more than that observed in the CR group (69\%), and the degree of negative heterosis for growth traits in the $\mathrm{RC}$ group was higher than that in the CR group. These results implied that the magnitude of the heterotic response was related to the proportion of genes with nonadditive expression.

To better understand the molecular basis of negative heterosis, functional enrichment analysis was performed to gain insight into the biological relevance of nonadditive inheritance in reciprocal crosses. We found that the process of oxidative phosphorylation was significantly enriched in nonadditive genes of reciprocal crosses, indicating the special and crucial roles of energy metabolism in the negative heterosis of growth traits. Several previous studies have described the correlation of oxidative phosphorylation with heterosis in corn [49], wheat [50] and rice [12]. Seymour et al. [2] found that the growth-related traits of Arabidopsis hybrids were associated with energy production via oxidative phosphorylation. This association was also reported in animals. McDaniel and Grimwood [51] demonstrated 




that heterosis of body weight in Drosophila melanogaster was correlated with oxidative phosphorylation efficiency. To validate the role of oxidative phosphorylation in negative heterosis of muscle yield in chickens, we further detected the ATP content and ATPase activity of breast muscle tissues for reciprocal crosses and parental lines. ATPase, as an essential enzyme in energy metabolism, catalyzes the hydrolysis of ATP to form ADP and harnesses the energy released from the breakdown of the phosphate bond to perform other cellular reactions. Our results showed that chickens with lower breast 


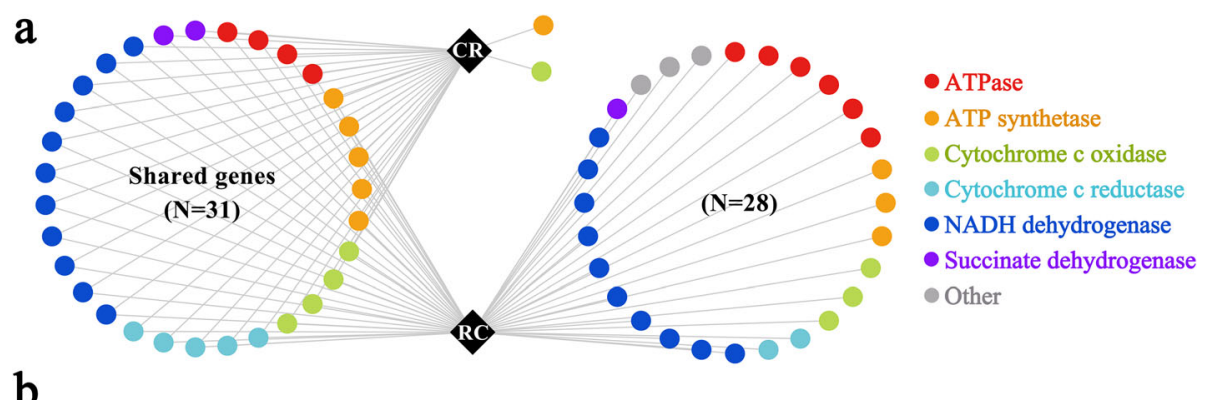

b

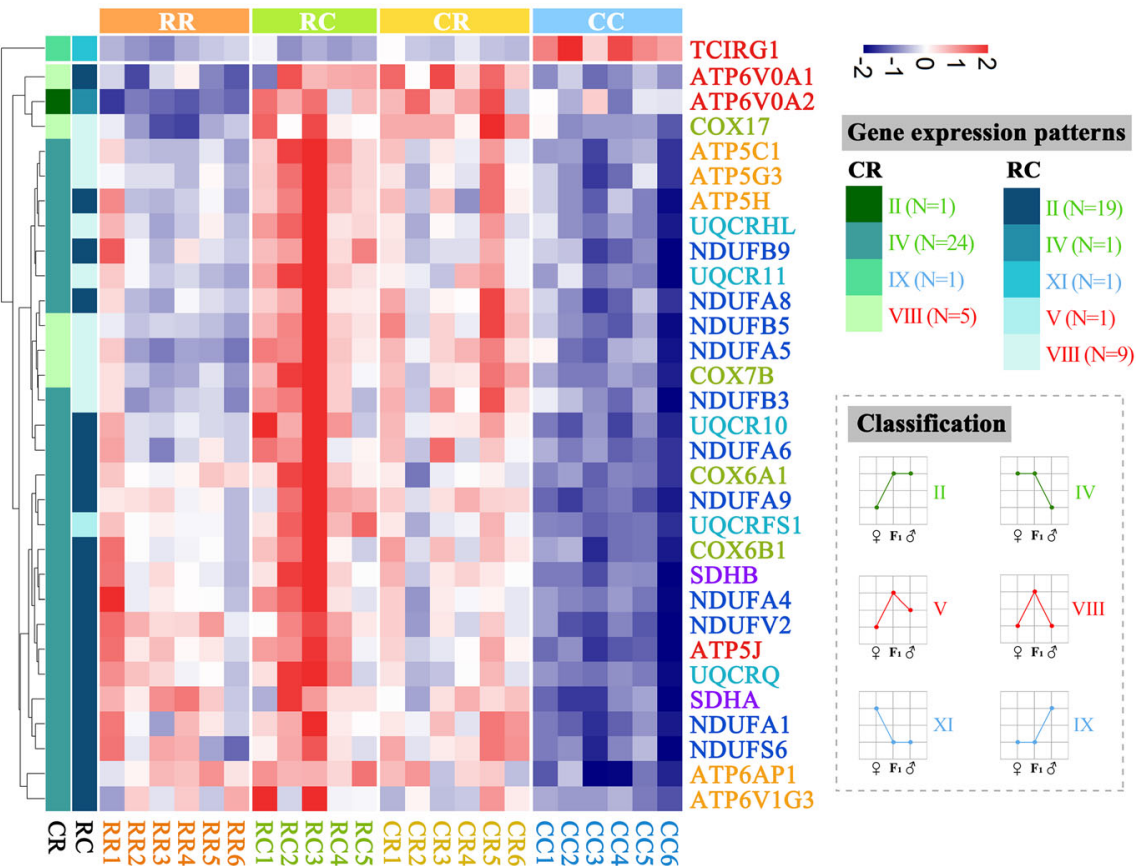

Fig. 5 Analyses of nonadditive genes enriched in oxidative phosphorylation of reciprocal crosses. a Overlap of nonadditive genes enriched in oxidative phosphorylation in the CR and RC groups. Each dot represents one gene. The names of the enzymes encoded by nonadditive genes are listed on the right. $\mathbf{b}$ Heatmap of shared gene expression levels in reciprocal crosses and their parents. The types of gene expression patterns in the $C R$ and $R C$ are shown on the left side. Schematic diagrams of the expression patterns and the number of genes are shown on the right. The color of the gene names represents the encoded enzyme, which is the same as the enzyme in plot (a)

muscle weight had lower ATP content but higher ATPase activity, suggesting that chickens with higher ATP consumption had lower meat production. This finding corroborated that energy metabolism contributed strongly to negative heterosis and might help provide effective strategies for reducing the rate of ATP hydrolysis to improve muscle yield in crossbreds. Since the expression level of nonadditive genes involved in oxidative phosphorylation of reciprocal progenies biased to the $\mathrm{R}$ line (egg-type chicken), the expression level in the $\mathrm{R}$ line was significantly higher than that in the $\mathrm{C}$ line. Thus, the objective of reducing the rate of ATP hydrolysis might be achieved by decreasing the difference in parental weights or increasing the proportion of broiler parentage in the crossbred population.
Growth is a complex polygenetic trait. To identify significant genes underlying the observed negative heterosis, we extracted the nonadditive genes detected in the process of oxidative phosphorylation in reciprocal crosses. A total of 31 shared genes were detected in reciprocal crosses. These genes encoding NADH dehydrogenase, cytochrome c reductase, cytochrome c oxidase, ATP synthase, ATPase and succinate dehydrogenase were all reported to be involved in the regulation of muscle growth and development, such as ATP5C1 [52], ATP5G3 [53], ATP5H [54, 55], ATP5J [53], ATP6AP1 [56], COX6B1 [57], NDUFA1 [52], NDUFA4 [52], NDUFA5 [52], NDUFA6 [52], NDUFV2 [53, 58], NDUFS6 [53], UQCR10 [52], UQCR11 [52], UQCRFS1 [57], SDHA [52, 53, 57] and SDHB [52, 53, 58]. Among these shared genes in reciprocal crosses, more than $60 \%$ 

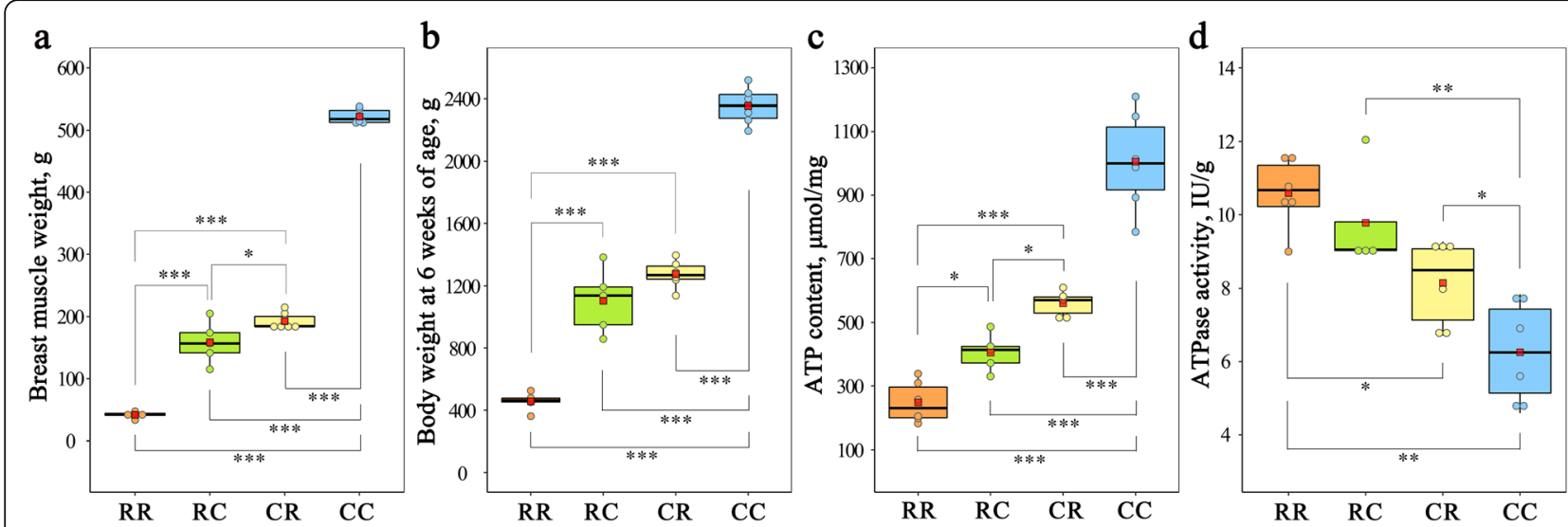

Fig. 6 ATP content and ATPase activity detection. Difference analysis of breast muscle weight (a), body weight (b), ATP content (c), and ATPase activity (d) among RR, RC, CR, and CC. For a-d, each dot represents a sample. The central red dot represents the mean value of the corresponding group. ${ }^{* *},{ }^{* *}$ and ${ }^{*}$ indicate adjusted $P$-values less than $0.001,0.01$, and 0.05 , respectively

of nonadditive genes exhibited a similar expression pattern to the layer line. The growth rate and body weight of layer chickens are considerably lower than those of broilers. It might be the large disparity of growth between layers and broilers and the differences in resource allocations that led to negative heterosis of growth traits in crossbred progenies. The Galgal5 may not be optimal chicken genome reference due to GRCg6a is available now, but should be sufficient to draw a conclusion that the important role of nonadditive genes and their related oxidative phosphorylation in negative heterosis for growth traits in chickens, since we confirmed the results by the detection of ATP content and ATPase activity. However, Gene expression is a dynamic process [59], and our research focused on gene expression analysis in juvenile chickens. We expect to determine whether the contributions of nonadditive genes would persist over time and to what degree they would impact the heterosis of growth traits in future experiments. In addition, the negative heterosis of growth traits in males was similar to that observed in females. However, sex-linked factors $[60,61]$, such as hormones, may influence the growth rate. Therefore, further experiments should be performed to confirm that nonadditive genes and their related oxidative phosphorylation are also the major genetic and molecular factors in the negative heterosis of growth in males.

\section{Conclusions}

Our research focused on the phenomenon of heterosis in chickens and found that negative heterosis of growth traits was more common than positive heterosis, especially for muscle yield. Whole genome-wide gene expression pattern analysis showed that nonadditivity was the major mode of gene action in crossbred chickens. Nonadditive genes related to the biological process of oxidative phosphorylation played a critical role in the formation of negative heterosis for growth traits. Chickens with higher ATP consumption had lower muscle production. Our study revealed fundamental mechanisms of negative heterosis for growth traits in chickens and has important implications for muscle yield improvement.

\section{Supplementary Information}

The online version contains supplementary material available at https://doi. org/10.1186/s40104-021-00574-2.

Additional file 1: Figure S1. Correlation among body weights of females and males at different ages. Figure S2. Correlation among body weight and muscle mass at 6 weeks of age. Figure S3. Volcano plot of differentially expressed genes between reciprocal crosses and parental lines. Figure S4. KEGG pathway analysis of overdominant genes in reciprocal crosses.

Additional file 2: Table S1. Descriptive statistics for body weight of females and males from hatch to 8 weeks of age. Table S2. Descriptive statistics for the left shank and sternum length of females and males during the experiment. Table S3. Summary statistics for transcriptome sequencing data. Table S4. Classification of different expression patterns of genes. Table S5. Descriptive statistics for heterosis of the length of shank and sternum for females and males during the experiment. Table S6. Heterosis of carcass performance for $F_{1}$ progenies at 6 weeks of age. Table S7. Gene expression patterns of differentially expressed genes in reciprocal crosses. Table S8. GO terms of the top 20 overdominant genes in the RC and CR groups. Table S9. Detailed information on the overlap of nonadditive genes in RC and CR groups.

\section{Abbreviations}

C: Cornish; CC: The purebred progenies that used Cornish as parents; CR: The crossbred progenies that used Cornish as the paternal line and Rhode Island White as the maternal line; DEGs: Differentially expressed genes;

FPKM: Fragments per kilobase million; GO: Gene Ontology; H\%: Heterosis as a percentage; KEGG: Kyoto Encyclopedia of Genes and Genomes; R: Rhode Island White; RC: The crossbred progenies that used Rhode Island White as the paternal line and Cornish as the maternal line; RR: The purebred progenies that used Cornish as parents; PCA: Principal component analysis; QRT-PCR: Quantitative real-time PCR 


\section{Acknowledgments}

We thank Dr. Guiqin Wu and Beijing Huadu Yukou Poultry Industry Co., Ltd., for providing the experimental chickens.

\section{Authors' contributions}

NY, CS and CW were involved in the conception of the study as well as the study design. CM and CW carried out the experiments. All authors participated in phenotypic and sample collection. CM, CW, NY and CS conducted bioinformatics and statistical analysis. CM detected the ATP content and ATPase activity of parental lines and reciprocal crosses. CM and CW designed all of the figures and wrote the manuscript. NY and CS were responsible for critical revisions of the manuscript drafts. All authors read and approved the final manuscript.

\section{Funding}

This work was supported by the National Natural Science Foundation of China (No. 31930105), China Agriculture Research Systems (CARS-40) and China Postdoctoral Science Foundation (No. 2020 M680028).

\section{Availability of data and materials}

The RNA sequencing data are available from the Sequence Read Archive (https://www.ncbi.nlm.nih.gov/sra) with BioProject number PRJNA524721.

\section{Declarations}

\section{Ethics approval and consent to participate}

This study was carried out obeying the Guidelines for Experimental Animals established by the Animal Care and Use Committee of China Agricultural University.

\section{Consent for publication}

Not applicable.

\section{Competing interests}

The authors declare that they have no conflict of interest.

Received: 22 October 2020 Accepted: 21 February 2021 Published online: 18 April 2021

\section{References}

1. Shull GH. The composition of a field of maize. J Hered. 1908;4:296-301.

2. Seymour DK, Chae E, Grimm DG, Martín Pizarro C, Habring-Müller A, Vasseur $F$, et al. Genetic architecture of nonadditive inheritance in Arabidopsis thaliana hybrids. Proc Natl Acad Sci U S A. 2016:113:E7317-26.

3. Davenport CB. Degeneration, albinism and inbreeding. Science. 1908;28: 454-5.

4. Jones DF. Dominance of linked factors as a means of accounting for heterosis. Proc Natl Acad Sci U S A. 1917:3:310-2.

5. East EM. Inbreeding in corn. Rep Conn Agric Exo Stn. 1907;1908:419-28.

6. Minvielle F. Dominance is not necessary for heterosis: a two-locus model. Genet Res. 1987:49:245-7.

7. Schnell FW, Cockerham CC. Multiplicative vs. arbitrary gene action in heterosis. Genetics. 1992:131:461-9.

8. Birchler JA, Auger DL, Riddle NC. In search of the molecular basis of Heterosis. Plant Cell. 2003:15:2236-9.

9. Hochholdinger $\mathrm{F}$, Hoecker $\mathrm{N}$. Towards the molecular basis of heterosis. Trends Plant Sci. 2007;12:427-32.

10. Skelly DA, Ronald J, Akey JM. Inherited variation in gene expression. Annu Rev Genom Hum G. 2009:10:313-32.

11. Wei G, Tao Y, Liu G, Chen C, Luo R, Xia H, et al. A transcriptomic analysis of superhybrid rice LYP9 and its parents. Proc Natl Acad Sci U S A. 2009;106: 7695-701.

12. Katara JL, Verma RL, Parida M, Ngangkham U, Molla KA, Barbadikar KM, et al. Differential expression of genes at panicle initiation and grain filling stages implied in heterosis of rice hybrids. Int J Mol Sci. 2020;21:1080.

13. Fujimoto R, Taylor JM, Shirasawa S, Peacock WJ, Dennis ES. Heterosis of Arabidopsis hybrids between C24 and col is associated with increased photosynthesis capacity. Proc Natl Acad Sci U S A. 2012:109:7109-14.

14. Hedgecock D, Lin J, DeCola S, Haudenschild CD, Meyer E, Manahan DT, et al. Transcriptomic analysis of growth heterosis in larval Pacific oysters (Crassostrea gigas). Proc Natl Acad Sci U S A. 2007;104:2313-8.
15. Moritsu Y, Nestor KE, Noble DO, Anthony NB, Bacon WL. Divergent selection for body weight and yolk precursor in Coturnix coturnix japonica. 12 Heterosis in reciprocal crosses between divergently selected lines. Poult Sci. 1997:76:437-44.

16. Rezvannejad E, Pakdel A, Ashtianee SRM, Yeganeh HM, Yaghoobi MM. Analysis of growth characteristics in short-term divergently selected Japanese quail lines and their cross. J Appl Poult Res. 2013;22:663-70.

17. Arthur PF, Makarechian M, Price MA, Berg RT. Heterosis, maternal and direct effects in double-muscled and normal cattle: II. Carcass traits of young bulls. J Anim Sci. 1989;67:911-9.

18. Bougas B, Granier S, Audet C, Bernatchez L. The transcriptional landscape of cross-specific hybrids and its possible link with growth in brook charr (Salvelinus fontinalis Mitchill). Genetics. 2010:186:97-107.

19. Clasen JB, Norberg E, Madsen P, Pedersen J, Kargo M. Estimation of genetic parameters and heterosis for longevity in crossbred Danish dairy cattle. J Dairy Sci. 2017;100:6337-42.

20. Weigel D, Bomblies K. Hybrid necrosis: autoimmunity as a potential geneflow barrier in plant species. Nat Rev Genet. 2007;8:382-93.

21. Bomblies K, Lempe J, Epple P, Warthmann N, Lanz C, Dangl JL, et al. Autoimmune response as a mechanism for a Dobzhansky-Muller-type incompatibility syndrome in plants. PLoS Biol. 2007:5:e236.

22. Ma Q, Hedden P, Zhang Q. Heterosis in rice seedlings: its relationship to gibberellin content and expression of gibberellin metabolism and signaling genes. Plant Physiol. 2011;156:1905-20.

23. Chen C, Chen H, Shan J, Zhu M, Shi M, Gao J, et al. Genetic and physiological analysis of a novel type of interspecific hybrid weakness in rice. Mol Plant. 2013;6:716-28.

24. East EM. Heterosis. Genetics. 1936:21:375-97.

25. Williams SM, Price SE, Siegel PB. Heterosis of growth and reproductive traits in fowl. Poult Sci. 2002:81:1109-12.

26. Jull MA, Quinn JP. The inheritance of body weight in the domestic fowl. J Hered. 1931;22:283-94.

27. Maw AJG. The inheritance of skeletal dimensions in the domestic fowl. Sci Agrár. 1935:16:85-112.

28. Liu G, Dunnington EA, Siegel PB. Maternal effects and heterosis for growth in reciprocal cross populations of chickens. J Anim Breed Genet. 1993;110: $423-8$.

29. Sutherland DAT, Honaker CF, Dorshorst B, Andersson L, Brisbin IJ, Siegel PB. Growth patterns for three generations of an intercross between red junglefowl and chickens selected for low body weight. J Anim Breed Genet 2018. https://doi.org/10.1111/jbg.12336.

30. Sun D, Wang D, Zhang Y, Yu Y, Xu G, Li J. Differential gene expression in liver of inbred chickens and their hybrid offspring. Anim Genet. 2005;36: 210-5.

31. Havenstein GB, Ferket PR, Qureshi MA. Carcass composition and yield of 1957 versus 2001 broilers when fed representative 1957 and 2001 broiler diets. Poult Sci. 2003:82:1509.

32. Swanson-Wagner RA, Jia Y, DeCook R, Borsuk LA, Nettleton D, Schnable PS. All possible modes of gene action are observed in a global comparison of gene expression in a maize $F_{1}$ hybrid and its inbred parents. Proc Natl Acad Sci U S A. 2006:103:6805-10.

33. Wu X, Li R, Li Q, Bao H, Wu C. Comparative transcriptome analysis among parental inbred and crosses reveals the role of dominance gene expression in heterosis in Drosophila melanogaster. Sci Rep. 2016;6:21124.

34. Mai C, Wen C, Sun C, Xu Z, Chen S, Yang N. Implications of gene inheritance patterns on the heterosis of abdominal fat deposition in chickens. Genes. 2019;10:824

35. Wu ZX, Zhang WC. Heterosis and statistical tests. Hereditas (Beijing). 1983;5: $24-6$.

36. Kim D, Langmead B, Salzberg SL. HISAT: a fast spliced aligner with low memory requirements. Nat Methods. 2015;12:357-60.

37. Pertea M, Pertea GM, Antonescu CM, Chang TC, Mendell JT, Salzberg SL. StringTie enables improved reconstruction of a transcriptome from RNA-seq reads. Nat Biotechnol. 2015:33:290-5.

38. El-Gebali S, Mistry J, Bateman A, Eddy SR, Luciani A, Potter SC, et al. The Pfam protein families database in 2019. Nucleic Acids Res. 2019:47:D427-32.

39. Liao Y, Smyth GK, Shi W. featureCounts: an efficient general purpose program for assigning sequence reads to genomic features. Bioinformatics. 2014;30:923-30.

40. Love Ml, Huber W, Anders S. Moderated estimation of fold change and dispersion for RNA-seq data with DESeq2. Genome Biol. 2014;15:550. 
41. Yu G, Wang L, Han Y, He Q. clusterProfiler: an R package for comparing biological themes among gene clusters. OMICS. 2012;16:284-7.

42. Du L, Chai D, Zhao L, Li X, Zhang F, Zhang H, et al. AMPK activation ameliorates Alzheimer's disease-like pathology and spatial memory impairment in a streptozotocin-induced Alzheimer's disease model in rats. J Alzheimers Dis. 2015;43:775-84.

43. Huang X, Yang S, Gong J, Zhao Q, Feng Q, Zhan Q, et al. Genomic architecture of heterosis for yield traits in rice. Nature. 2016;537:629-33.

44. Shao L, Xing F, Xu C, Zhang Q, Che J, Wang X, et al. Patterns of genomewide allele-specific expression in hybrid rice and the implications on the genetic basis of heterosis. Proc Natl Acad Sci U S A. 2019;116:5653-8.

45. Yang J, Mezmouk S, Baumgarten A, Buckler ES, Guill KE, McMullen MD, et al. Incomplete dominance of deleterious alleles contributes substantially to trait variation and heterosis in maize. PLoS Genet. 2017;13:e1007019.

46. Sutherland DAT, Honaker CF, Dorshorst B, Andersson L, Siegel PB. Asymmetries, heterosis, and phenotypic profiles of red junglefowl, white Plymouth rocks, and F1 and F2 reciprocal crosses. J Appl Genet. 2018;59: 193-201.

47. Li X, Wei Y, Nettleton D, Brummer EC. Comparative gene expression profiles between heterotic and non-heterotic hybrids of tetraploid Medicago sativa. BMC Plant Biol. 2009;9:107.

48. Li A, Fang MD, Song WQ, Chen CB, Qi LW, Wang CG. Gene expression profiles of two intraspecific Larix lines and their reciprocal hybrids. Mol Biol Rep. 2012;39:3773-84.

49. McDaniel RG, Sarkissian IV. Heterosis: complementation by mitochondria. Science. 1966:152:1640-2.

50. Sarkissian IV, Srivastava HK. High efficiency, heterosis, and homeostasis in mitochondria of wheat. Proc Natl Acad Sci U S A. 1969;63:302-9.

51. McDaniel RG, Grimwood BG. Hybrid vigor in Drosophila: respiration and mitochondrial energy conservation. Comp Biochem Physiol B Comp Biochem. 1971;38:309-14.

52. Wu C, Satomi Y, Walsh K. RNA-seq and metabolomic analyses of AKT1mediated muscle growth reveals regulation of regenerative pathways and changes in the muscle secretome. BMC Genomics. 2017;18:181.

53. Liu X, Du Y, Trakooljul N, Brand B, Muráni E, Krischek C, et al. Muscle transcriptional profile based on muscle fiber, mitochondrial respiratory activity, and metabolic enzymes. Int J Biol Sci. 2015;11:1348-62.

54. Chazarin B, Storey KB, Ziemianin A, Chanon S, Plumel M, Chery I, et al. Metabolic reprogramming involving glycolysis in the hibernating brown bear skeletal muscle. Front Zool. 2019;16:12.

55. Potts JK, Echternkamp SE, Smith TPL, Reecy JM. Characterization of gene expression in double-muscled and normal-muscled bovine embryos. Anim Genet. 2003;34:438-44.

56. Lee EJ, Kamli MR, Pokharel S, Malik A, Tareq KM, Roouf BA, et al. Expressed sequence tags for bovine muscle satellite cells, myotube formed-cells and adipocyte-like cells. PLoS One. 2013;8:e79780.

57. Barbe C, Bray F, Gueugneau M, Devassine S, Lause P, Tokarski C, et al. Comparative proteomic and transcriptomic analysis of follistatin-induced skeletal muscle hypertrophy. J Proteome Res. 2017;16:3477-90.

58. Hong J, Kim BW, Choo HJ, Park JJ, Yi JS, Yu DM, et al. Mitochondrial complex I deficiency enhances skeletal myogenesis but impairs insulin signaling through SIRT1 inactivation. J Biol Chem. 2014;289:20012-25.

59. Bar-Joseph Z, Gitter A, Simon I. Studying and modelling dynamic biological processes using time-series gene expression data. Nat Rev Genet. 2012;13: 552-64.

60. Barbato GF, Vasilatos-Younken R. Sex-linked and maternal effects on growth in chickens. Poult Sci. 1991;70:709-18.

61. Gibson G, Riley-Berger R, Harshman L, Kopp A, Vacha S, Nuzhdin S, et al. Extensive sex-specific nonadditivity of gene expression in Drosophila melanogaster. Genetics. 2004;167:1791-9.

Ready to submit your research? Choose BMC and benefit from:

- fast, convenient online submission

- thorough peer review by experienced researchers in your field

- rapid publication on acceptance

- support for research data, including large and complex data types

- gold Open Access which fosters wider collaboration and increased citations

- maximum visibility for your research: over $100 \mathrm{M}$ website views per year

At BMC, research is always in progress.

Learn more biomedcentral.com/submissions 